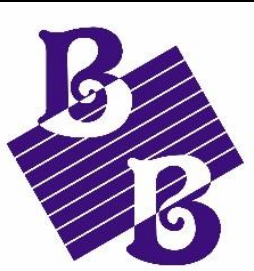

Biobacta
Journal of Bioscience and Applied Research WWW.JBAAR.ORG

\title{
The validity of the Questionnaire to identify Schistosoma haematobium infection
}

\author{
Ahmed Abdelhalim Yameny \\ Society of Pathological Biochemistry and Hematology, Egypt \\ Ahmed A. Yameny (Email: dr.ahmedyameny@yahoo.com)
}

DOI: $10.21608 /$ jbaar.2017.126468

\begin{abstract}
Background: Schistosomiasis is considered the most important water-based disease, Estimation of S.haematobium infection has been recognized in Egypt since ancient times, hematuria known as AAA disease. One of the symptoms of urinary schistosomiasis is visible blood in urine, since this is normally well recognized by children, the assessment of urinary schistosomiasis can be undertaken rapidly and accurately by using a simple questionnaire. This study was done to test the validity of self-reported blood in urine by questionnaire compared to parasitological methods. Subjects and methods: Across -sectional study was carried. To overall total sample was 1000 patients of different ages, data about the history of recent symptoms of schistosomiasis as haematuria and dysuria were collected. Also, urine microscopic examination was done for the detection of S.haematobium eggs by sedimentation centrifugation and nuclepore filtration techniques. Results: The results showed that haematuria by questionnaire had a sensitivity of $54.4 \%$ and specificity of $90.8 \%$. When dysuria was considered, sensitivity increased to $75.95 \%$ but specificity decreased to $51.2 \%$. When both self-reported haematuria and dysuria were considered, sensitivity was $68.3 \%$ and specificity $85 \%$, in between the previous results. PPVs ranged between $11.8 \%$ and $33.6 \%$ whereas NPVs ranged between $95.9 \%$ and $96.1 \%$. As for diagnostic efficiency, it was best for haematuria (87.9\%).
\end{abstract}

Conclusion: questionnaire has a moderate sensitivity, a simple questionnaire asking "Did you have blood in urine during the last month?" and "Did you suffer from schistosomiasis during the last months?" is a validated tool that allows for a decision to be taken, a questionnaire was shown to have a very high negative predictive value $96 \%$, so that it is safe to use it to identify communities with a low prevalence so that they can be excluded from the first phases of a control program, but microscopic examination is better and not expensive for diagnosis of urinary schistosomiasis infection.

Keywords: Schistosoma haematobium, questionnaire, self-reported, validity, Schistosoma eggs, urinary schistosomiasis.

Received: October 20, 2017. Accepted: December 20, 2017. Published: December 22, 2017 


\section{Introduction}

Schistosomiasis is considered the most important water-based disease ${ }^{(1)}$. Many species of schistosomes can infect humans, but the most important from the medical point of view are; S.mansoni, S.japonicum, and S.haematobium ${ }^{(2)}$. Estimation of S.haematobium infection has been recognized in Egypt since ancient times, hematuria known as AAA disease was mentioned in the Ebris the papyri, remedies for hematuria were recorded in papyri ${ }^{(3,4)}$. Egypt started to fight against the schistosome disease in 1915, after the discovery of the schistosome life cycle, using snail control and chemotherapy $^{(5)}$. Due to these control projects, the prevalence of S.mansoni decreased from $32 \%$ in 1935 to less than $0.2 \%$ in 2016 , and the prevalence of S.haematobium from $48 \%$ in 1935 to less than $0.2 \%$ in 2016 , but in 300 villages the prevalence still more than 3\%, so the Ministry of Health and Population in Egypt has started a campaign to reach the final elimination of schistosomiasis by $2020^{(6)}$.

Urinary schistosomiasis infection depends on water contact activities with some risk factors as gender, age, residence, education, and occupation so the control program should be done upon to health education on these risk factors with snail control and chemotherapy ${ }^{(7)}$. In areas where data on schistosomiasis are completely absent, the parasitological survey might not be sufficient to evaluate correctly the parasitological situation. To better estimate the prevalence of schistosomiasis, given its focal distribution and its linkage to water resources, it will be necessary to associate the parasitological survey with the Rapid Assessment Method (RAM) that is based on questionnaires ${ }^{(8)}$. One of the symptoms of urinary schistosomiasis is visible blood in urine, since this is normally well recognized by children, the assessment of urinary schistosomiasis can be undertaken rapidly and accurately by using a simple questionnaire distributed to all schools in a delimited administrative or geographical area, the method has proved effective for ranking schools according to the level of transmission of urinary schistosomiasis (WHO1995) ${ }^{\left({ }^{9}\right)}$. The school rank obtained with RAM will allow for a better interpretation of the parasitological results since only high (or low) prevalence schools could be selected by chance ${ }^{(10)}$. The number of children presenting visible blood in urine must be registered because this is an indicator of heavy infection ${ }^{(11)}$. If a person shows visible haematuria, in an area endemic for S.haematobium, there is no need for further diagnostic investigation of that person ${ }^{(10)}$.

The questionnaire is a rapid means of identifying heavily infected schools that should be treated first in urinary schistosomiasis control. It should be sent to all schools in the area concerned, which can be done at a relatively low cost, the questionnaire is short and simple and asks the children whether they have seen blood in their urine at any time during the past month (or a shorter period) ${ }^{(9)}$. In a school where the prevalence of infection is $40 \%$, half of the infected children would not report schistosomiasis, whereas, in a school where the prevalence was $80 \%$, only a quarter of infections would not have been reported. This observation has important practical relevance for the application of mass treatment when the prevalence of infection exceeds $50 \%$. If the prevalence of self-reported schistosomiasis underestimates the actual prevalence of infection by $20 \%$, then mass treatment may be given in schools where the prevalence of self-reported schistosomiasis is $>30$ $\%{ }^{(12)}$. Prevalence of reported schistosomiasis in schools of $>30 \%$ is equivalent to a prevalence of infection of $>50 \%$ and that mass treatment would be warranted (WHO 1995). However, responses may be affected by the age and gender, by the interviewer, and by an awareness of the aims of the program and the expectation of free treatment. The present study used both questionnaire and microscopic examination of urine samples to detect S.haematobium eggs for 1000 patient samples to evaluate the validity of the questionnaire compared 
with a microscopic examination for the diagnosis of S.haematobium infection.

\section{Research objective:}

To evaluate the validity of the questionnaire compared with a microscopic examination for the diagnosis of S.haematobium infection.

\section{Material and methods}

\subsection{Study population and ethical consideration}

This study included 1000 patients attending the Ministry of health laboratory centers, in ElFayoum Governorate, these study subjects were randomly selected irrespective of the age-group and both genders were included. All the studied population were informed about the purpose of samples collection and their consents were obtained. Patients were free to refuse sample collection.

\subsection{Study design}

This research is a cross-sectional study designed to evaluate a questionnaire of self-reported blood in the urine to determine urinary schistosomiasis in the study population. This study was targeting customers who came to laboratories of health centers for urine analysis.

\subsection{Collection and processing of urine samples}

Clean specimen bottles were labeled with the needed information and issued to the participating individuals whose informed consent was sought earlier, each patient was given a wide mouth screw-capped container into which to void urine. This was carried out between 10.00 am and 2.00 pm when the ova count of S.haematobium is expected to be at its peak ${ }^{(13)}$. In this study, urinary schistosomiasis was defined as the presence of ova of S.haematobium in the urine.

\subsection{Urine microscopy}

Urine examination was carried out in the laboratory of health centers and by the researcher.
Urine samples were examined for the presence of S.haematobium eggs as in the sedimentation method of Cheesbrough (2006) ${ }^{(14)}$. Each urine sample was mixed thoroughly with a glass rod and two samples were taken for each $10 \mathrm{ml}$ urine, one sample for sedimentation centrifugation and the other $10 \mathrm{ml}$ urine sample for the Nuclepore membrane filtration technique. The first $10 \mathrm{ml}$ transferred into a centrifuge tube and centrifuged at $2000 \mathrm{rpm}$ for 5 minutes at room temperature. The supernatant was then discarded and sediment was transferred to a microscope glass slide and covered with a coverslip. A drop of Lugol's Iodine was added onto the coverslip before the examination. Examination of the entire sediment was carried out using the $\mathrm{x} 10$ objective of a compound light microscope.

The second $10 \mathrm{ml}$ urine sample was examined using the Nuclepore membrane filtration technique for S.haematobium eggs detection as in the method of Cheesbrough (2009) ${ }^{(15)}$.

\subsection{Questionnaire:}

For individuals attending health laboratories, the following was done:-

Completing a predesigned interview questionnaire that covered the following:

1-Sociodemographic data:- age, sex, occupation, residence, etc-----

2-Exposure to canal water whether, occupational, recreational, or domestic exposure.

3-Health problems in the previous week for example burning micturition, urinating blood, malaise, Schistosoma infection, and menstruation in females.

4-History of hematuria or dysuria

\subsection{Statistical analysis}


Results collected, coded, tabulated, and analyzed through computer facilities using statistical methods S.haematobium infection was defined as any number of eggs greater than zero found in 10 $\mathrm{ml}$ of urine, was performed to compare with selfreported haematuria by simple questionnaire for Diagnosis of Schistosoma haematobium Infection.

\section{Results}

In this study, we considered using the sum of Nuclepore membranes filtration technique and Centrifugation sedimentation technique results as a gold standard to compare them with the Questionnaire of self-reported Haematuria and Dysuria $^{(16)}$.

Table (1): shows a diagnostic performance of selfreported haematuria, dysuria, and both as diagnostic methods for Schistosoma haematobium infection compared to microscopic examination techniques as the gold standard.

The results showed that haematuria by questionnaire had a sensitivity of $54.4 \%$ and specificity of $90.8 \%$. When dysuria was considered, sensitivity increased to $75.95 \%$ but specificity decreased to $51.2 \%$. When both self reported haematuria and dysuria were considered, sensitivity was $68.3 \%$ and specificity $85 \%$, in between the previous results. PPVs ranged between $11.8 \%$ and $33.6 \%$ whereas NPVs ranged between $95.9 \%$ and $96.1 \%$. As for diagnostic efficiency, it was best for haematuria $(87.9 \%)$ followed by both haematuria and dysuria $83.36 \%$, and least for dysuria $(53.1 \%)$. The area under the curve (AUC) was sufficient for haematuria and dysuria( 0.68 and 0.6 respectively) and was good for $\operatorname{both}(0.72)$.

Table (1): Diagnostic performance of self reported haematuria, dysuria and both as diagnostic methods for schistosoma haematobium compared to microscopic examination techniques as the gold standard.

\begin{tabular}{|c|c|c|c|c|c|c|c|c|c|c|c|c|}
\hline \multirow{2}{*}{\multicolumn{2}{|c|}{ Test }} & \multicolumn{2}{|c|}{$\begin{array}{c}\text { Microscopic } \\
\text { techniques }\end{array}$} & \multirow{2}{*}{$\begin{array}{r}\text { Total } \\
\text { No. }\end{array}$} & \multirow[t]{2}{*}{$\begin{array}{c}\text { Sensitivity } \\
\%\end{array}$} & \multirow[t]{2}{*}{$\begin{array}{c}\text { Specificity } \\
\%\end{array}$} & \multirow{2}{*}{$\begin{array}{c}\text { PPV } \\
\%\end{array}$} & \multirow{2}{*}{$\begin{array}{c}\text { NPV } \\
\%\end{array}$} & \multirow{2}{*}{$\underset{\%}{\text { PLR }}$} & \multirow{2}{*}{$\begin{array}{c}\text { NLR } \\
\%\end{array}$} & \multirow{2}{*}{$\begin{array}{c}\text { Diagnostic } \\
\text { Efficiency } \\
\%\end{array}$} & \multirow[t]{2}{*}{ AUC } \\
\hline & & $\begin{array}{c}\text { Negative } \\
\text { No. }\end{array}$ & $\begin{array}{c}\text { Positive } \\
\text { No. }\end{array}$ & & & & & & & & & \\
\hline \multirow[t]{3}{*}{$\mathrm{HU}$} & No & 836 & 36 & 872 & \multirow[t]{3}{*}{54.4} & \multirow[t]{3}{*}{90.8} & \multirow[t]{3}{*}{33.6} & \multirow[t]{3}{*}{95.9} & \multirow[t]{3}{*}{5.9} & \multirow[t]{3}{*}{0.5} & \multirow[t]{3}{*}{87.90} & \multirow[t]{3}{*}{0.68} \\
\hline & Yes & 85 & 43 & 128 & & & & & & & & \\
\hline & Total & 921 & 79 & 1000 & & & & & & & & \\
\hline \multirow[t]{3}{*}{ Dysuria } & No & 472 & 20 & 492 & \multirow{3}{*}{75.95} & \multirow{3}{*}{51.2} & \multirow{3}{*}{11.8} & \multirow{3}{*}{96.1} & \multirow{3}{*}{1.53} & \multirow{3}{*}{0.49} & \multirow{3}{*}{53.1} & \multirow{3}{*}{0.60} \\
\hline & Yes & 449 & 59 & 508 & & & & & & & & \\
\hline & Total & 921 & 79 & 1000 & & & & & & & & \\
\hline \multirow{3}{*}{$\begin{array}{l}\text { both } \\
\text { haematuria } \\
\text { and dysuria }\end{array}$} & No & 470 & 19 & 489 & \multirow[t]{3}{*}{68.3} & \multirow[t]{3}{*}{85} & \multirow[t]{3}{*}{33.1} & \multirow[t]{3}{*}{96.1} & \multirow[t]{3}{*}{4.55} & \multirow[t]{3}{*}{0.37} & \multirow[t]{3}{*}{83.36} & 0.72 \\
\hline & Yes & 83 & 41 & 124 & & & & & & & & \\
\hline & Total & 553 & 60 & 613 & & & & & & & & \\
\hline
\end{tabular}

PPXPositive predictive value; NPP, Negative predictive value; PLR, Likelihood ratio for positive results; NPL Likelihood ratio for negative results; 


\section{Discussion}

For S. haematobium infection, the presence of micro-or macro-haematuria has enabled the development and validation of a range of indirect diagnostic tests useful for epidemiological mapping of prevalence, such as dipstick methods which detect micro-and macrohaematuria. Simple interview methods to ascertain a history of haematuria have also been shown to be useful. For example, the WHO-supported red urine study and others have established the utility of a simple oral questionnaire for a history of haematuria, to estimate the prevalence of infection among school-age children ${ }^{(17,18)}$. When urgent intervention is required to prevent death and liver on bladder damage, often in an environment of civil unrest or man-made or natural disaster, any extra labor and costs needed are problematic. Although no new alternative, simple, fast screening method has been produced, scientific efforts have validated the usefulness of the questionnaire and the reagent strip as simple tools to estimate Schistosoma haematobium infection ${ }^{(19)}$. Therefore a simple questionnaire asking "Did you have blood in urine during the last month?" and "Did you suffer from schistosomiasis during the last months?" is a validated tool that allows for a decision to be taken. The decision to treat all school-age children when $30 \%$ of them reported gross haematuria, indicating that $50 \%$ have detectable eggs in urine. (Guyatt et al., 1999) ${ }^{(17)}$.

Lower predictive values are observed for the entire population, especially for women of childbearing age due to confounding conditions such as menstruation, pregnancy, and genitourinary infections (Utzerger et al., 1998) ${ }^{(20)}$. This aspect must be taken into consideration when planning the complementary strategy of the deworming program which is based on community action and treatment of groups at high risk of infection. A matter of concern is that the efficiency of the questionnaire and strips test decreases as the prevalence and intensity of infection decrease ${ }^{(19)}$. The "indirect interview" approach using questionnaires, is a method for diagnosis urinary schistosomiasis at a community level. It is based on the hypothesis that if communities are ranked according to the percentage of positive answers to the questions "Did you have blood in your urine?" or "Did you have schistosomiasis?" the rank order will be the same as that obtained by ranking them according to the actual prevalence of schistosomiasis, the questionnaire approach first had to be validated to check that this hypothesis is true, by comparing the results for the new approach with those obtained with standard tests ${ }^{(8)}$.

This study showed that self-reported schistosomiasis for the presence of blood in the urine (microhematuria) has a sensitivity of $54.4 \%$, a specificity of $90.8 \%$, a positive predictive value of $33.6 \%$, and a negative predictive value of $95.87 \%$, while the sensitivity of dysuria increased to $76 \%$ but its specificity decreased to $51.2 \%$ when both self reported haematuria and dysuria sensitivity was $68.3 \%$ and specificity $85 \%$ in between the previous results, PPVs ranged between $11.8 \%$ and $33.6 \%$, whereas NPVs ranged between $95.9 \%$ and $96.1 \%$, the diagnostic efficiency it was best for haematuria $87.9 \%$, followed by both haematuria and dysuria $83.4 \%$ and lower for dysuria $53.1 \%$. The area under the curve (AUC) was sufficient for haematuria and dysuria (0.68 and 0.6 respectively) and was good for both (0.72).

The questionnaire method for determining communities at high risk for urinary schistosomiasis was validated in some African countries, Cameron, Congo, Ethiopia, Malawi, Tanzania, Zambia, and Zimbabwe ${ }^{(21,22)}$. The prevalence of urinary schistosomiasis obtained using the questionnaire survey was compared with the prevalence obtained using urine filtration and/or testing for haematuria with chemical reagent strips. In all but one country (Ethiopia) the 
method was found to work well for "community diagnosis ". It was shown to have a very high negative predictive value so that it is safe to use it to identify communities with a low prevalence so that they can be excluded from the first phases of a control program and resources concentrated elsewhere. A few communities with only moderate or low prevalences may not be excluded, because the questionnaires do not have $100 \%$ accuracy, but including a few communities which do not need intervention is less dangerous than missing some that do ${ }^{(21,22)}$.

Ansel J, et al., (1997) studied the reliability of self-reported blood in urine and schistosomiasis as indicators of Schistosoma haematobium infection in Tanzania, they found that the prevalence of reported schistosomiasis showed a strong correlation with prevalence assessed by the microscopy of urine. The sensitivity was ranged from $50 \%$ to $75 \%$ according to the heaviness of infection and the specificity was ranged from $66.4 \%$ to $96.4 \%{ }^{(12)}$.

Lwambo et al., (1997) concluded that medical history alone is not suitable for identifying infected individuals because the sensitivity can be as low as $40 \%$ for a history of bloody urine among adults ${ }^{(23)}$.

Guyatt $\mathrm{H}$ et al., (1999), reported that pain on urination and blood in urine showed strong specificity but very poor sensitivity compared to microscopic egg counts, it must be remembered that these reported symptoms can be caused by other urinary tract infection ${ }^{(17)}$.

Pogggeness G, et al., (2000) studied the validity of self-reported symptoms for a screening of Tanzanian women of childbearing age for urinary schistosomiasis. They found that blood in urine has a sensitivity of $34.9 \%$, a specificity of 85.2 , a positive predictive value in the low-risk site was $9.1 \%$, a positive predictive value in the high-risk site was $52.7 \%$, negative predictive value in low-risk site way $96.3 \%$ and negative predictive value in the high-risk site was $50 \%{ }^{(24)}$.

MD et al French., (2007). Recorded that these questionnaire tests showed considerable inter-year variability, it seems that they are not reliable general indicators that track the dynamics of morbidity ${ }^{(25)}$.

Fen Yang et al, (2015). The study for the validity of the questionnaire showed that; diagnostic odds ratio (DOR) was obtained from Schistosoma haematobium (67.68, $95 \%$ confidence interval $(\mathrm{CI})=31.48 \quad$ to145.54), sensitivity $96 \%$, specificity $86 \%$, AUC was $0.96^{(26)}$.

Howeida H. Abusalih, et al., (2016). Recorded that Percentage of hematuria in the study population by questionnaire hematuria against the presence of Schistosoma eggs in urine revealed sensitivity, specificity, positive and negative predictive values as $55.6 \%, 80 \%, 43 \%$, and $86.9 \%$ respectively ${ }^{(27)}$.

\section{Conflict of interest}

There are no conflicts of interest.

\section{Financial support and sponsorship}

This research did not receive any specific grant from funding agencies in the public, commercial, or not-for-profit sectors.

\section{References:}

1. Steinmann P, Keiser J, Bos R, Tanner M, Utzinger J. Schistosomiasis, and water resources development: a systematic review, meta-analysis, and estimates of people at risk. Lancet Infect Dis.2006; 6(7): 411-25.

2. Gryseels B, Polman K, Clerinx J, Kestens L. Human schistosomiasis. Lancet. 2006; 368(9541):1106-18. 
3. Mobark AB. The schistosomiasis problem in Egypt. Am J Trop Med Hyg 1982; 31(1): 8791.

4. Miller RL, Armelagos GJ, Ikram S, de Janger N, Krijger FW, Deeler AM. Paleoepidemiology of Schistosoma infection in mummies. Br Med J 1992; 304: 555-6.

5. Youssef MM. Current situation and research needs for schistosomiasis control in Egypt (2005), Geneva, WHO Tor/ SWG/ 01, 2005.

6. Ahmed A. Yameny. The evolving schistosomiasis agenda 2017- 2020 in Egypt: Moving from control toward final elimination. J Biosci App Res .2017; 3(2): 48- 54.

7. Ahmed A. Yameny. Schistosomiasis haematobium prevalence and risk factors in El-Fayoum Governorate, Egypt. J Biosci App Res. 2017;3(3):191-201.

8. Chitsulo L, Lengeler C, and Jenkins J.The schistosomiasis manual: A guide for the rapid identification of communities with a high prevalence of urinary schistosomiasis (1995); UNDP/World Bank/WHO Special Programme for Research \& Training in Tropical Diseases (TDR).

9. WHO (2002): Helminth control in school-age children a guide for managers of control programs, Geneva.

10. WHO (1998): Guidelines for the evaluation of soil-transmitted helminthiasis and schistosomiasis at a community level, Geneva WHO/ CDS/ Sip/ 98.1.

11. Savioli L, Hatz C, Dixon H, Kisumkuu UM, Mott KE. Control of morbidity due to $\mathrm{S}$. haematobium on Pemba Island: egg excretion and haematuria as indicators of infection. Am J Trop Med Hyg 1990; 43: 289- 295.

12. Ansel J, Guyatt H, Hall A, Kihamiq C, Kivugo $\mathrm{J}$, Ntimbwq $\mathrm{P}$, et al. The reliability of selfreported blood in urine and Schistosoma haematobium infection in school children: a study in Muhenza District, Tanzania. Tropical
Medicine and International Health 1997; 2(12): 80-89.

13. Eyong ME, Ikepeme EE, Ekanem EE. Relationship between Schistosoma haematobium infection and urinary tract infection among children in South Eastern, Nigeria. Niger Postgrad Med J2008; 15:89-93.

14. Cheesbrough M. District laboratory practice in tropical countries part 2. United Kingdom: Cambridge University Press. Cambridge. 2006; 323-41.

15. Cheesbrough M. Laboratory-practice-intropical-countries-part-1. United Kingdom: Cambridge University Press. Cambridge. 2009;p.357

16. Ahmed A. Yameny. Evaluation of Nuclepore Filteration Technique for Diagnosis of Schistosoma haematobium Infection. J Biosci App Res. 2015;1(6):335-341.

17. Guyatt H, Brooker S, Lwambo NJ, Siza JE, Bundy DA (1999) The performance of schoolbased questionnaires of reported blood in urine in diagnosing Schistosoma haematobium infection: patterns by age and sex. Trop Med Int Health 4: 751-757.

18. WHO (1998) Report of the WHO informal consultation on schistosomiasis control. Geneva.

19. Rabello A, and Enk M. Progress towards the detection of schistosomiasis .report of schistosomiasis working group on schistosomiasis, 2005, pp.67-71.

20. Utzinger $\mathrm{J}$ et al. Schistosoma mansoni, intestinal parasites and perceived morbidity indicators in school children in a rural endemic area of western Cote d'Ivoire. Tropical Medicine and International Health, 1998, 3: 711 - 720.

21. Lengeler C, Kilima P, Mshinda H, Morona D, Hatz C, Tanner M.(1991a); Rapid, low-cost, two-step method to screen for urinary schistosomiasis at the district level: the Killosa 
experience. Bulletin of the World Health Organization 69:179 - 189 .

22. Red Urine Study Group (1995). Identification of high-risk communities for schistosomiasis in Africa: A multi-country study. WHO TDR/SER Report Series, in press.

23. Lwambo NJ et al. Control of Schistosoma haematobium morbidity on Pemba Island: validity and efficiency of indirect screening tests. Bulletin of the World Health Organization, 1997, 75:247-252.

24. G. Poggensee, L. Krantz, I. Kiwelu, H. Feldmeier. Screening of Tanzanian women of childbearing age for urinary schistosomiasis: validity of urine reagent strip readings and self-reported symptoms. Bulletin of the World Health Organization, 2000, 78(4).

25. French MD, Rollinson D, Basanez M, Mgeni AF, Khames IS, and Stothard R. Schol-based control of urinary schistosomiasis on Zanzibar,
Tanzania: Monitoring micro-haematuria with reagent strips as a rapid urological assessment. Journal of Pediatric Urology volume 3, issue5, October 2007, pages 364-368.

26. Fen Yang, Xiao-dong Tan, Bei Liu1, Chongming Yang3, Zi-ling Nil, Xu-dong Gao, and Ying Wang. A meta-analysis of the diagnostic efficiency of the questionnaires screening for schistosomiasis. Parasitol Res (2015) 114:3509-3519.

27. Howeida H. Abusalih, Mohamed A. Awadelkarim, Elsadig Yousif Mohamed, and Sawsan Mustafa Abdalla. Rapid screening of Urinary Schistosomiasis using simple Questionnaire: East Nile locality, Khartoum state, Sudan. European Journal of Pharmaceutical and Medical Research, 2016, 3(3), 56-59. 